\title{
Clinical Trials Corner Issue 7(4)
}

Piyush K. Agarwal ${ }^{\mathrm{a}}$ and Cora N. Sternberg ${ }^{\mathrm{b}}$

${ }^{a}$ The University of Chicago, Chicago, IL, USA

${ }^{\mathrm{b}}$ Englander Institute for Precision Medicine, Weill Cornell Medicine, Meyer Cancer Center, New York, NY, USA

Received 4 November 2021

Accepted 8 November 2021

Pre-press 15 November 2021

Published 13 December 2021

Dear Readers,

In this issue, we highlight several trials presented at this year's European Society for Medical Oncology (ESMO) annual meeting. These trials evaluate chemotherapy and targeted local therapy . In the future, please reach out to us directly in order to highlight any specific clinical trials at pkagarwal@uchicago.edu or cns9006@med.cornell.edu and/or at BLC@iospress.com.

Sincerely,

Piyush K. Agarwal, MD

Associate Editor, Bladder Cancer

Director, Bladder Cancer Program

The University of Chicago

Chicago, Illinois

\section{Cora N. Sternberg, MD, FACP}

Associate Editor, Bladder Cancer

Clinical Director, Englander Institute for Precision Medicine

Weill Cornell Medicine

New York, New York

Study Title: Randomized Phase III Study of Gemcitabine/Cisplatine (GC) Versus High-dose Intensity Methotrexate, Vinblastine, Doxorubicine and Cisplatin (HD-MVAC) in the Perioperative Setting for Patients With Locally Advanced Transitional Cell Cancer of the Bladder

\section{Clinicaltrials.gov identifier: NCT 01812369}

Sponsor: University Hospital, Rouen

\section{Enrollment: 500}

Rationale: Gemcitabine and cisplatin are commonly used for perioperative (neoadjuvant or adjuvant) chemotherapy based on data demonstrating similar efficacy with better tolerance as compared to MVAC (methotrexate, vinblastine, doxorubicin, and cisplatin) in metastatic disease. In metastatic disease, highdose (HD)-MVAC has better efficacy and this trial evaluates whether HD-MVAC has better efficacy in the perioperative setting in terms of progression-free survival.

Study Design: The GETUG/AFU V05 VESPER Phase III Trial randomized trial was conducted at 28 French centers comparing 4 cycles of gemcitabine and cisplatin (GC) to 6 cycles of dose dense or high-dose MVAC in the perioperative treatment of muscle-invasive bladder cancer (MIBC). Treatment was given either in the neoadjuvant or adjuvant window. Patients with pure or mixed urothelial bladder cancer were enrolled but neuroendocrine histology was excluded. All patients were platinum-eligible. 
Endpoints: The primary endpoint was progression-free survival (PFS) at three years.

Results: Overall, 437 patients (88\%) received neoadjuvant chemotherapy: $84 \%$ of the patients in the GC arm and $60 \%$ of the patients in the HD-MVAC arm received all planned cycles. $90 \%$ and $91 \%$ of the patients underwent surgery respectively and organ-confined disease (<ypT3N0) was more frequently seen in the HDMVAC arm (77\% vs. $63 \%, \mathrm{p}=0.001)$ respectively. The adjuvant group was smaller (only 54 patients) with $81 \%$ of the patients in the GC arm and $40 \%$ of the patients in the HD-MVAC arm receiving all planned cycles. Overall, the PFS at three years was better in the HD-MVAC arm (64\% vs. 56\%, HR $=0.77$ (95\% CI, 0.57-1.02), $\mathrm{p}=0.066)$ in all patients (neoadjuvant and adjuvant) but it was significantly better in the neoadjuvant group $(66 \%$ vs. $56 \%, \mathrm{HR}=0.70(95 \% \mathrm{CI}, 0.51-0.96), \mathrm{p}=0.025)$. OS data are not mature yet and final analysis will be done after a median follow-up of 5 years.

Comments: The trial demonstrated a better organ-confined rate and a better PFS at 3 years in the HD-MVAC arm. Among the planned cycles in each arm of the trial, fewer patients in the HD-MVAC arm were treated with all planned cycles compared to the GC arm. Despite that, the cystectomy rate was the same in both arms suggesting that if a patient can tolerate this neoadjuvant regimen, it may result in better local control and progression-free survival. This regimen should be considered as a new standard for fit patients with muscle invasive urothelial cancer. The small number of patients treated with adjuvant chemotherapy prevent any conclusions about these regimens given on an adjuvant basis.

Reference: ESMO Abstract \#6520: VESPER (GETUG/AFU V05): A phase III trial of Dose dense Methotrexate, Vinblastine, Doxorubicin and Cisplatin (dd-MVAC) or Gemcitabine and Cisplatin (GC) as perioperative chemotherapy for patients with muscle-invasive bladder cancer (MIBC). Christian Pfister et al.

\section{Study Title: A Phase 1b-2 Study to Evaluate Safety, Efficacy, Pharmacokinetics, and Pharmacodynamics of Various Regimens of Erdafitinib in Subjects With Metastatic or Locally Advanced Urothelial Cancer}

\section{Clinicaltrials.gov identifier: NCT03473743}

Sponsor: Janssen Research \& Development, LLC

\section{Enrollment: 126}

Rationale: Currently, first-line therapy for cisplatin-ineligible patients with metastatic urothelial cancer is alternative chemotherapy or anti-PD-(L)-1 monotherapy. The pan-fibroblast growth factor receptor (FGFR) inhibitor, erdafitinib, is indicated as second-line therapy in metastatic urothelial cancer with targetable FGFR alterations. The phase Ib trial determined a tolerable dose of erdafitinib and the anti-PD-1 antibody, cetrelimab, as a second-line regimen in metastatic patients. The phase II part of the trial is reported here which evaluates erdafitinib alone or erdafitinib and cetrelimab as a first-line regimen in cisplatin-ineligible patients with FGFR alterations.

Study Design: Patients with metastatic urothelial cancer with select FGFR alterations, measurable disease, no prior systemic therapy, and who were ineligible to receive cisplatin were enrolled. Any PD-(L)-1 status could be enrolled. Patients were randomized to either erdafitinib alone or erdafitinib and cetrelimab.

Endpoints: The primary endpoints were overall response rate (ORR) per RECIST 1.1 and safety. Secondary endpoints included disease control rate, time to response, and duration of response.

Results: The reported results of the Norse Trial at ESMO 2021 were as of July 2021. As of that date, 53 patients were randomized but ORR was evaluable in 37 patients. The ORR was $33 \%$ in the erdafitinib arm vs. 
$68 \%$ in the combination arm. The complete response rate was $6 \%$ vs. $21 \%$, respectively. The median duration of response was not evaluable in the erdafitinib arm and 6.9 months in the combination arm. The safety data included 48 total patients with the side effects being attributable to the ertafinitib including hyperphosphatemia ( $58 \%$ erdafitinib vs. $58 \%$ combination), stomatitis ( $63 \%$ vs. $54 \%$, respectively), and diarrhea (50\% vs. $42 \%$, respectively).

Comments: The results demonstrate activity with the combination of erdafitinib and cetrelimab in first line, cisplatin-ineligible patients with metastatic urothelial cancer with FGFR alterations. These alterations are more frequent in upper tract tumors than in bladder cancer. Although of interest, other very promising regimens for first line, cisplatin-ineligible patients currently exist (such as enfortumab vedotin and pembrolizumab).

Reference:https://oncologypro.esmo.org/meeting-resources/esmo-congress-2021/erdafitinib-erda-or-erda-pluscetrelimab-cet-for-patients-with-metastatic-or-locally-advanced-urothelial-carcinoma-muc-and-fibroblastgrowth

\section{Study Title: A Phase II Trial of sEphB4-HSA in Combination With Anti PD1 Antibody Pembrolizumab (MK-7435) for Metastatic Urothelial Cancer Refractory to Platinum}

\section{Clinicaltrials.gov identifier: NCT02717156}

Sponsor: University of Southern California

\section{Enrollment: 60}

Rationale: EphrinB2 (B2) is expressed in metastatic urothelial cancer and it blocks immune cell traffic into the tumor. B2 can be blocked by EphrinB4 (B4) which can theoretically increase immune cell traffic and sEphB4HSA is a fusion protein of soluble EphrinB4 and albumin that binds to EphrinB2. This trial evaluates the combination of pembrolizumab in combination with sEphB4-HSA in previously treated metastatic urothelial cancer with the hypothesis that an influx of immune cells would enhance the response to a anti-PD-(L)-1 therapy.

Study Design: Patients with platinum-refractory metastatic urothelial cancer and no prior PD-(L)-1 therapy were treated with the combination of sEphB4-HSA and pembrolizumab in a single-arm, single-center Phase II study. Treatment was continued until progression or unacceptable toxicity and response was measured every 6 weeks using RECIST 1.1. Tumor tissue for all evaluable patients was tested for B2 expression by immunohistochemistry.

Endpoints: The primary endpoint was overall survival (OS) while secondary endpoints were progression free survival (PFS), duration of response (DOR), and objective response rate (ORR).

Results: 69 patients were enrolled. The main sites of disease were the nodes, lungs, and the liver. The median overall survival was 14.4 months. The ORR was $38 \%$ with a median PFS of 4.0 and a median DOR of 8 months. Expression of B2 was seen in $46(65.7 \%)$ patients. ORR was $57.5 \%$ among B2+ patients with a DOR of 27 months. Among the toxicities, hypertension was the most common attributable to B4. Other toxicities include fatigue, nausea, headache, anorexia, proteinuria, hyperuricemia, rash, anemia, and elevated AST. The percentage of grade 3-4 toxicities seen was $42 \%$.

Comments: This trial demonstrated impressive results with combination therapy that was more pronounced in those patients with EphrinB2 expression. The combination has received breakthrough designation by the FDA. 
Given the mechanism of action, it will be interesting to see whether the fusion protein will be synergistic with other immune therapies such as CTLA-4 inhibitors or possibly even BCG.

Reference: ESMO Abstract \#6510: Phase II trial of pembrolizumab (P) in combination with sEphB4-HSA (B4) in previously treated metastatic urothelial carcinoma (mUC). Sarmad Sadeghi et al.

\section{DISCLOSURES}

\section{Cora N. Sternberg}

Consultant: Pfizer, Merck Ga, AstraZeneca, Astellas Pharma, Sanofi-Genzyme, Roche/Genentech, Medscape, Immunomedics, Clovis Oncology, UroToday, MSD, Foundation Medicine

\section{Piyush K. Agarwal}

Advisory Board (unpaid): AstraZeneca; Advisory Board (paid): AURA, Verity, UROGEN, Janssen 